\title{
Influência da composição da biomassa no rendimento em condensáveis da torrefação de resíduos vegetais
}

\author{
Lucélia Alves de Macedo ${ }^{1}$ Patrick Louis Albert Rousset², Ailton Teixeira do Vale ${ }^{3}$ \\ ${ }^{1}$ Serviço Florestal Brasileiro - SCEN, Trecho 02, Edifício Sede do IBAMA, Asa Norte, CEP 70.818-900, Brasília, DF, Brasil \\ ${ }^{2}$ King Mongkut's of Technology Thonburi, Chatuchak, 10900, Bangkok, Thaïland \\ ${ }^{3}$ Universidade de Brasília, Campus Universitário Darcy Ribeiro, CEP 70.910-900, Brasília, DF, Brasil
}

*Autor correspondente:

luceliamac@yahoo.com.br

Termos para indexação:

Composição química

Tratamento térmico

Análise imediata

Index terms:

Chemical composition

Thermal treatment

Liquid by-product

Histórico do artigo:

Recebido em 14/07/2014

Aprovado em 24/10/2014

Publicado em 31/12/2014

doi: $10.4336 / 2014 . p f b .34 .80 .747$
Resumo - O objetivo do trabalho foi avaliar a influência da composição da biomassa (teores de cinzas, materiais voláteis, carbono fixo, carbono, hidrogênio, oxigênio, lignina, holocelulose e extrativos) no rendimento em condensáveis da torrefação de casca de arroz (Oryza sativa L.), da casca de pinhão-manso (Jatropha curcas L.), do capim elefante (Pennisetum purpureum Schum. var. mineiro), do bagaço de cana (Saccharum officinarum L.) e do bambu (Bambusa vulgaris ex J.C. Wendl. var. vulgaris). As biomassas, com partículas entre $0,5 \mathrm{~mm}$ e $1,0 \mathrm{~mm}$, foram submetidas à torrefação em um gradiente de temperatura variando de $250{ }^{\circ} \mathrm{C}$ a $300^{\circ} \mathrm{C}$, taxa de aquecimento de $20^{\circ} \mathrm{C} \min ^{-1}$ e tempo de residência de 15 minutos. Foram realizados 5 ensaios por biomassa e obtidos os rendimentos em líquido, sólido e gás. O teor de holocelulose e o teor de voláteis das biomassas apresentaram correlação positiva e significativa com o rendimento em condensáveis. O teor de cinzas apresentou correlação negativa e significativa com o rendimento em condensáveis. Não houve correlação significativa entre a composição química elementar e o rendimento em condensáveis.

\section{Effect of biomass composition on the condensable gas yield from torrefaction of plant residues}

\begin{abstract}
This work assessed the effect of biomass composition (ash, volatile matter and fixed carbon content, carbon, hydrogen and nitrogen content, lignin, extractives and holocellulose content) on the condensable gas yield from the torrefaction of rice husk (Oryza sativa L.), jatropha seed husk (Jatropha curcas L.), elephant grass (Pennisetum purpureum Schum. var. mineiro); sugarcane bagasse (Sacharum officinarum L.) and bamboo (Bambusa vulgaris ex J.C. Wendl. var. vulgaris). Biomasses with a particle size between $0.5 \mathrm{~mm}$ and $1.0 \mathrm{~mm}$ were subjected to torrefaction process using a temperature gradient varying from $250{ }^{\circ} \mathrm{C}$ to $300^{\circ} \mathrm{C}$, for $15 \mathrm{~min}$, with a heating rate of $20^{\circ} \mathrm{C} \mathrm{min}{ }^{-1}$. Five trials were conducted for each biomass and solid, liquid and gas yields were obtained. The holocellulose and the volatile matter content of biomass showed a positive and significant correlation with condensable yield. The ash content showed a negative and significant correlation with condensable yield. There was no significant correlation between the elementary chemical composition and the condensable yield.
\end{abstract}




\section{Introdução}

Os problemas globais associados ao uso intensivo de combustíveis fósseis aumentaram nos últimos anos o interesse por processos termoquímicos de conversão de biomassa em combustíveis, produtos químicos e outros materiais (Wild et al., 2009).

Materiais lignocelulósicos podem ser usados como matéria-prima para a produção comercial dos mesmos tipos de produtos originários do petróleo, como diversos tipos de combustíveis, solventes e outros produtos químicos (Yaman, 2004). No Brasil, biomassas como resíduos de serraria, resíduos da exploração florestal e resíduos agrícolas constituem um enorme potencial para aproveitamento energético e valorização química.

Um dos processos termoquímicos de conversão que vem ganhando destaque como pré-tratamento da biomassa para fins energéticos é a torrefação, um processo de tratamento térmico que se desenvolve na fase endotérmica da pirólise, entre $200{ }^{\circ} \mathrm{C}$ e $300^{\circ} \mathrm{C}$, em atmosfera inerte (Prins et al., 2006; Almeida et al., 2010) onde a hemicelulose, fração mais reativa da biomassa $\mathrm{e}$ principal fonte de voláteis, é decomposta (Prins et al., 2006; Arias et al., 2008). O resultado deste processo é um material sólido intermediário entre a biomassa e o carvão (Felfli et al., 2000), muito utilizado em processos de gaseificação, além de voláteis condensáveis que podem servir de matéria-prima para produtos químicos (Tumuluru et al., 2011).

Os gases condensáveis (excluindo a água), que representam cerca de $8 \%$ do balanço global do processo de torrefação, tem despertado o interesse da indústria química para aproveitamento na síntese de produtos químicos. No entanto, o baixo rendimento da fração condensável representa um gargalo para a valorização química deste subproduto. $\mathrm{O}$ rendimento e a qualidade dos subprodutos da torrefação são influenciados pela composição da biomassa, tamanho da partícula, temperatura do processo, tempo de residência, taxa de aquecimento (Rodrigues \& Rousset, 2009; Medic et al., 2010) e do teor de oxigênio na atmosfera de trabalho (Rousset et al., 2012).

A maioria dos estudos sobre torrefação é voltada para a melhoria da qualidade do subproduto sólido (Rodrigues \& Rousset, 2009; Kim et al., 2012; Rousset et al., 2012; Wannapeera \& Worasuwannarak, 2012; Ohliger et al., 2013), além de estudos sobre a influência dos parâmetros operacionais no rendimento dos subprodutos do processo
(Prins et al., 2006; Wannapeera et al., 2011; Lee et al., 2012), com pouca ênfase sobre o impacto da composição da matéria-prima.

Com o recente interesse em direcionar o processo de torrefação para a produção de condensáveis, torna-se indispensável realizar estudos voltados para a otimização da produção desse subproduto, bem como avaliar a influência da composição da biomassa no rendimento, qualidade e quantidade dos gases condensáveis. O estudo desses fatores permitirão adaptações na matériaprima, melhorias no controle do processo, bem como otimização energética e valorização química dos coprodutos (Tumuluru et al., 2011).

Neste contexto, este estudo teve por objetivo avaliar a influência da composição da biomassa no rendimento em gases condensáveis durante o processo de torrefação de seis biomassas de rápido crescimento.

\section{Material e métodos}

Foram utilizadas biomassas de rápido crescimento de capim elefante (Pennisetum purpureum Schum var. mineiro) e bambu (Bambusa vulgaris ex J.C. Wendl. var. vulgaris), com 1 ano de idade, e das espécies bagaço de cana-de-açúcar (Saccharum officinarum L.), casca de arroz (Oryza sativa L.) e casca de pinhão-manso (Jatropha curcas L.)

\section{Determinação da composição da biomassa}

A análise imediata (teores de voláteis, cinza e carbono fixo) foi conduzida de acordo com a norma ABNT NBR8112 (Associação Brasileira de Normas Técnicas, 1986).

As determinações dos teores de carbono, hidrogênio e nitrogênio foram feitas no analisador elementar Vario Macro $\mathrm{CHN}$, de acordo com os procedimentos da norma europeia XP CEN/TS 15104 (European Committee for Standardezation, 2011) Os resultados foram obtidos em base seca e livre de cinzas. O teor de oxigênio foi obtido por diferença.

A preparação das amostras para determinação dos teores de lignina e extrativos foi feita de acordo com os procedimentos da norma TAPPI T-264 om-88 (Technical Association of the Pulp and Paper Industry, 1996c). Para obter amostras livres de extrativos (preparação necessária para a análise de lignina), bem como para determinar os teores de extrativos solúveis em etanol:tolueno, foram utilizadas as normas TAPPI T 204 om-88 (Technical Association of the Pulp and 
Paper Industry, 1996a) e TAPPI T-211 om-93 (Technical Association of the Pulp and Paper Industry, 1996b).

O conteúdo de lignina foi determinado de acordo com o método NREL LAP 003 descrito por Templeton \& Ehrman (1995) para lignina insolúvel e NREL LAP 004 descrito por Ehrman (1996), para lignina solúvel. As análises foram realizadas em amostras livres de extrativos. O teor de lignina total é a soma das frações solúvel e insolúvel. O teor de holocelulose foi obtido por diferença, subtraindo da massa inicial da amostra (livre de extrativos), o teor de lignina total e de cinzas.

\section{Reator de torrefação}

Os experimentos foram conduzidos em um reator de torrefação tubular de aço aquecido por resistências elétricas, provido de um sistema de condensação de gases. O equipamento é composto de dois tubos de aço, sendo que o externo é fechado na parte superior, possuindo uma abertura para entrada do termopar de controle.

O tubo interno possui um compartimento para acomodar a biomassa em sua porção superior, que consiste de uma placa microperfurada, permitindo a circulação de gases. A injeção de nitrogênio foi feita durante todo o processo, com fluxo de $0,1 \mathrm{~L} \mathrm{~min}^{-1}$, utilizando-se o controlador de fluxo Brooks, Série Smart, modelo 5850S. O sistema de condensação foi imerso em banho criogênico à cerca de $-3^{\circ} \mathrm{C}$. Os gases condensáveis ficaram retidos no condensador tipo serpentina e os gases não condensáveis foram eliminados pela saída lateral do condensador.

\section{Preparação das amostras para os ensaios de torrefação}

As amostras foram trituradas e classificadas de modo a se obter partículas entre $0,5 \mathrm{~mm}$ e $1,0 \mathrm{~mm}$. Para cada espécie, foram obtidas 5 amostras de biomassa de aproximadamente $3,5 \mathrm{~g}$. As amostras foram secas em estufa a $102 \pm 3{ }^{\circ} \mathrm{C}$, até massa constante à umidade de $0 \%$.

\section{Parâmetros do processo de torrefação}

Os ensaios foram conduzidos em um gradiente de temperatura varinado de $250{ }^{\circ} \mathrm{C}$ no topo do leito a $300{ }^{\circ} \mathrm{C}$ na base deste, com taxa de aquecimento de $20^{\circ} \mathrm{C} \mathrm{min}^{-1}$, durante 15 minutos, para todas as biomassas.

\section{Obtenção dos rendimentos dos subprodutos}

Os rendimentos dos subprodutos da torrefação (gases condensáveis totais, sólido torrificado e gases não condensáveis) foram reportados como um percentual da massa inicial seca da biomassa.
A massa de condensáveis foi obtida pela pesagem do condensador antes e após o experimento. O rendimento em condensáveis totais $(\mathrm{RC})$ foi obtido pela expressão 1 . O teor de água presente na fração condensável foi determinado de acordo com o método de titulação Karl Fisher.

$$
R C(\%)=\left(M C / M B_{0 \%}\right) \times 100
$$

Onde: $\mathrm{MC}=$ massa de condensáveis totais $(\mathrm{g})$;

$\mathrm{MB}_{0 \%}=$ massa seca inicial da biomassa $(\mathrm{g})$.

A massa do sólido torrificado foi obtida imediatamente após os ensaios. O rendimento em subproduto sólido (RS) foi obtido pela expressão 2.

$R S(\%)=\left(M B_{T} / M B_{0 \%}\right) \times 100$

Onde: $\mathrm{MB}_{\mathrm{T}}=$ massa de biomassa torrificada $(\mathrm{g})$;

$\mathrm{MB}_{0 \%}=$ massa seca inicial da biomassa $(\mathrm{g})$.

$O$ rendimento em gases não condensáveis $(R G)$ foi obtido por diferença, pela expressão 3 .

$$
R G=100-(R C+R S)
$$

\section{Correlações entre composição da biomassa e o rendimento em condensáveis}

Foi feita correlação de Pearson entre as variáveis, buscando encontrar correlações entre a composição da biomassa e o rendimento em condensáveis.

\section{Resultados e discussão}

\section{Composição da matéria-prima}

Os resultados referentes à composição das biomassas constam na Tabela 1.

Houve grande variação na composição das biomassas analisadas, quanto aos teores de cinzas, voláteis e carbono fixo, com todas as médias estatisticamente diferentes. Os teores de cinza variaram de $2,1 \%$ a $26,4 \%$ e os de voláteis de $55,9 \%$ a $78,3 \%$, respectivamente, para a casca de arroz e para o bambu. Os teores de carbono fixo variaram de $13,9 \%$ a $21,3 \%$, respectivamente, para o bagaço de cana e para a casca de pinhão-manso.

O teor de cinzas de uma biomassa pode variar em função de tratos culturais, de contaminações decorrentes do manuseio e armazenamento, além de diferenças relacionadas à idade e partes da planta. $\mathrm{O}$ menor teor de cinzas encontrado para o capim elefante, quando comparado aos $11 \%$ encontrados por Seye et al. (2000) e Pérez et al. (2002), pode ser atribuído à idade do material. O capim utilizado neste estudo apresentava um ano de idade e, neste caso, a razão colmo/folha é maior do que a encontrada em capins mais jovens, possuindo, portanto, menos cinzas, visto que o teor de cinzas nas folhas do capim é maior do que aquele encontrado no colmo (Vamvuka et al., 2010). 
Tabela 1. Valores médios dos teores de cinza (CZ), materiais voláteis (MV), carbono fixo (CF), carbono (C), hidrogênio (H), nitrogênio $(\mathrm{N})$ oxigênio $(\mathrm{O})$, lignina total $(\mathrm{LIG})$, extrativos solúveis em etanol tolueno (EXT) e holocelulose (HOL).

\begin{tabular}{l|ccc|ccccc|ccc}
\hline \multirow{2}{*}{ Biomassa } & \multicolumn{4}{|c|}{$\%$} & \multicolumn{4}{c|}{$\%$} & \multicolumn{3}{c}{$\%$} \\
\cline { 2 - 11 } & $\mathbf{C Z}$ & MV & $\mathbf{C F}$ & $\mathbf{C}$ & $\mathbf{H}$ & $\mathbf{N}$ & $\mathbf{O} * *$ & LIG & EXT & HOL ** \\
\hline Casca de arroz & $26,4 \mathrm{a}$ & $55,9 \mathrm{e}$ & $17,7 \mathrm{c}$ & $47,3 \mathrm{~d}$ & $6,1 \mathrm{~b}$ & $0,9 \mathrm{~b}$ & $45,7 \mathrm{a}$ & $27,0 \mathrm{a}$ & $1,6 \mathrm{e}$ & $55,83 \mathrm{c}$ \\
Bagaço de cana & $11,0 \mathrm{~b}$ & $75,0 \mathrm{c}$ & $13,9 \mathrm{e}$ & $48,1 \mathrm{c}$ & $5,9 \mathrm{c}$ & $0,5 \mathrm{c}$ & $45,5 \mathrm{a}$ & $24,9 \mathrm{ab}$ & $6,9 \mathrm{~b}$ & $70,31 \mathrm{~b}$ \\
Casca de pinhão-manso & $9,4 \mathrm{c}$ & $69,3 \mathrm{~d}$ & $21,3 \mathrm{a}$ & $50,8 \mathrm{a}$ & $6,5 \mathrm{a}$ & $1,5 \mathrm{a}$ & $41,3 \mathrm{c}$ & $26,0 \mathrm{a}$ & $7,8 \mathrm{a}$ & $70,26 \mathrm{~b}$ \\
Capim elefante & $5,2 \mathrm{~d}$ & $77,6 \mathrm{~b}$ & $17,2 \mathrm{~d}$ & $49,2 \mathrm{~b}$ & $6,1 \mathrm{~b}$ & $1,1 \mathrm{~b}$ & $43,6 \mathrm{~b}$ & $23,2 \mathrm{~b}$ & $6,2 \mathrm{c}$ & $74,15 \mathrm{a}$ \\
Bambu & $2,1 \mathrm{e}$ & $78,3 \mathrm{a}$ & $19,6 \mathrm{~b}$ & $49,6 \mathrm{~b}$ & $6,1 \mathrm{~b}$ & $0,4 \mathrm{c}$ & $44,0 \mathrm{~b}$ & $25,8 \mathrm{a}$ & $5,5 \mathrm{~d}$ & $73,67 \mathrm{a}$ \\
\hline
\end{tabular}

Médias seguidas pela mesma letra na coluna não diferem estatisticamente a $5 \%$ de probabilidade pelo teste de Tukey. * Base seca e livre de cinzas. ** Obtido por diferença.

As biomassas apresentaram composição química elementar, em base seca e livre de cinzas, semelhante a valores encontrados para madeiras, conforme Oliveira et al. (1982) que encontraram valores entre $40 \%$ a $50,5 \%$ de carbono, $34 \%$ a $45 \%$ de oxigênio, $4,8 \%$ a $7,0 \%$ de hidrogênio e valores abaixo de $1 \%$ para nitrogênio.

Houve pouca variação nos teores de lignina das biomassas, variando de $23,2 \%$ a $27 \%$, respectivamente para o capim elefante e casca de arroz. Os teores de extrativos variaram de $1,6 \%$ a $7,8 \%$, respectivamente para as cascas de arroz e as de pinhão-manso. $\mathrm{O}$ teor de holocelulose da casca de arroz é significativamente inferior aos das outras biomassas, fato este associado ao seu alto teor de cinzas. Os maiores teores de holocelulose foram encontrados para o bambu e capim elefante, que apresentaram os menores teores de cinzas. Essa relação entre teores de holocelulose e cinzas é mostrada na Figura 1.

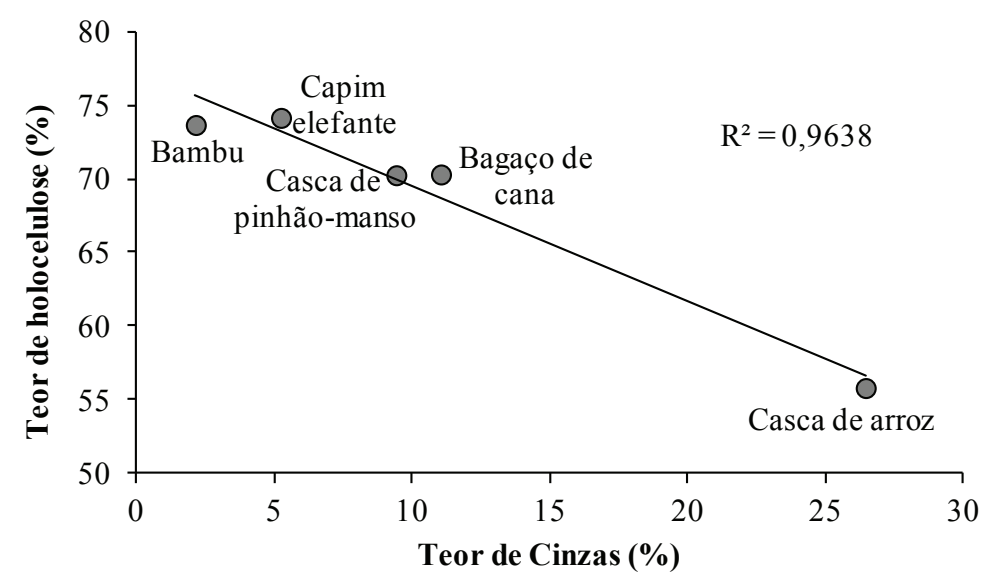

Figura 1. Relação entre o teor de holocelulose e o teor de cinzas das biomassas.

O fato de a lignina apresentar maior tendência à formação de subproduto sólido e a holocululose (celulose + hemiceluloses) tenderem a se decompor em produtos voláteis (Di Blasi, 1993) poderia explicar a alta correlação encontrada entre o teor de holocelulose e o teor de materiais voláteis das biomassas (Figura 2).

\section{Rendimentos dos subprodutos}

$\mathrm{Na}$ Figura 3 são apresentados os rendimentos dos produtos da torrefação para as biomassas analisadas, incluindo os gases condensáveis totais (líquidos orgânicos e água), o subproduto sólido e os gases não condensáveis.

Os rendimentos médios encontrados para os condensáveis totais, sólido e gases não condensáveis para as cinco biomassas foram $27,0 \%, 52,3 \%$ e $20,7 \%$, respectivamente. $\mathrm{O}$ rendimento em gases condensáveis variou de $20,2 \%$ a $32,7 \%$, respectivamente para a casca de arroz e bambu. Os rendimentos da casca de pinhãomanso, do capim elefante e do bagaço de cana não diferiram estatisticamente entre si. 


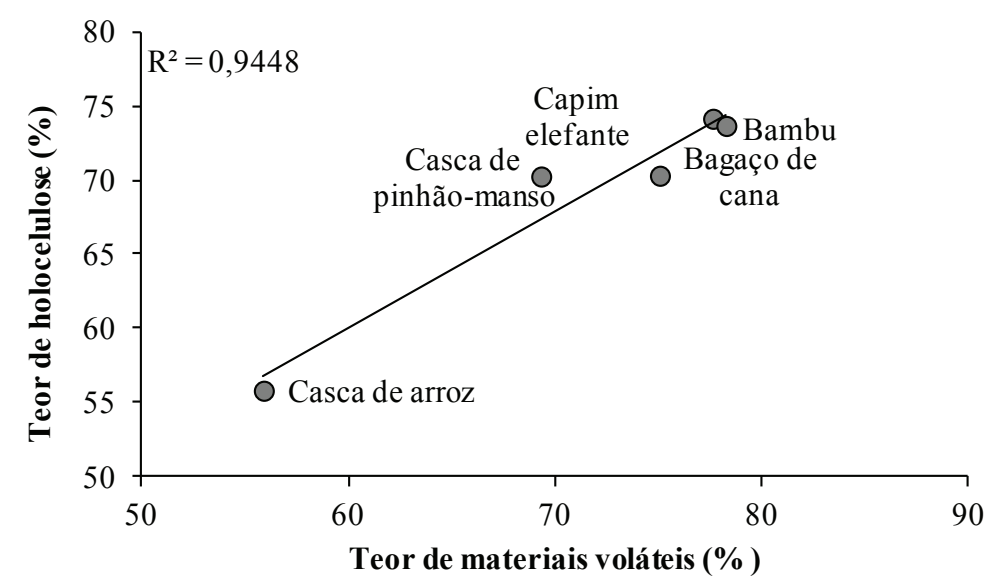

Figura 2. Relação entre os teores de holocelulose e materiais voláteis das biomassas.

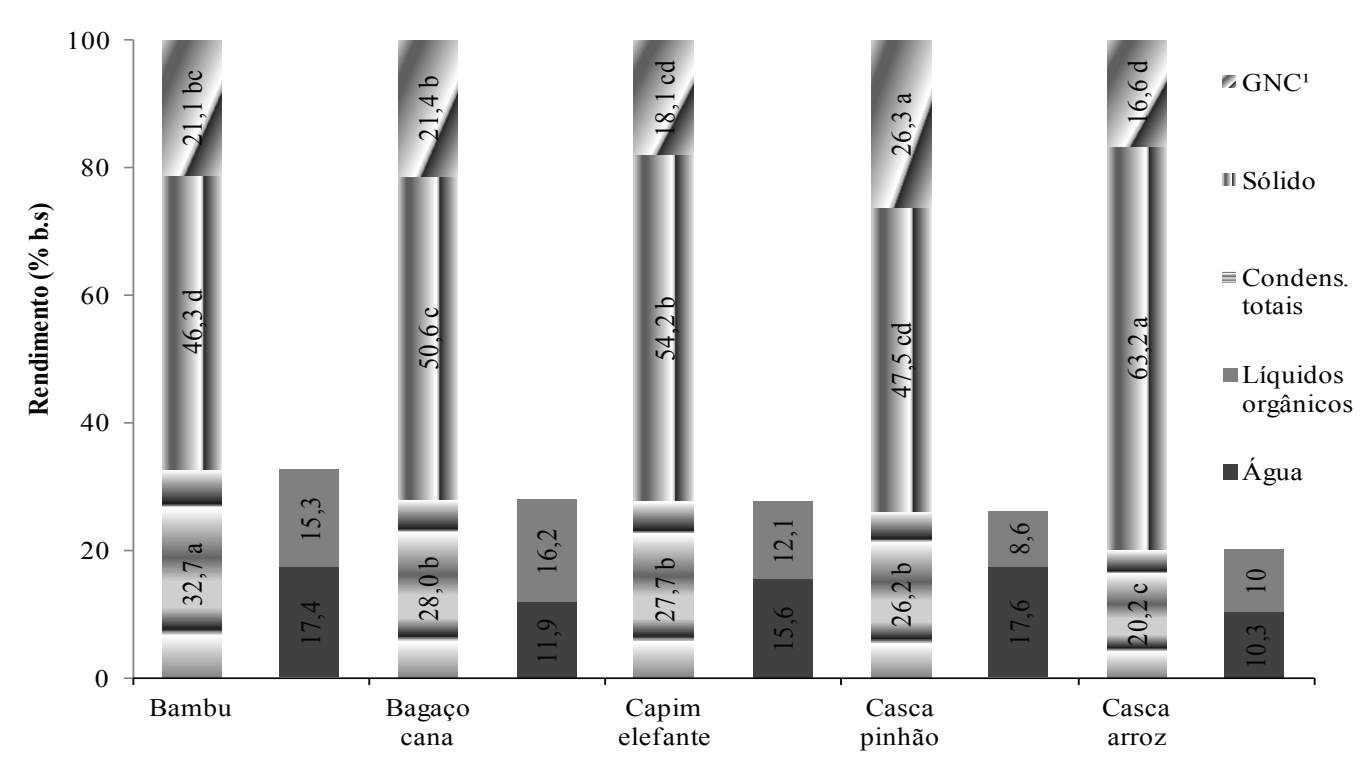

Figura 3. Rendimentos dos subprodutos da torrefação (base seca). GNC = gases não condensáveis. ${ }^{1}$ Obtido por diferença. Médias seguidas pela mesma letra em cada categoria não diferem estatisticamente a $5 \%$ de probabilidade, pelo teste de Tukey.

A maior perda de massa, com a consequente redução do rendimento em subproduto sólido e mais alto rendimento em condensáveis totais do bambu, também foi observada por Chen et al. (2011) estudando a torrefação de uma espécie de bambu e duas espécies de madeiras. Esses autores verificaram que o bambu apresentou maior taxa de degradação, independente da temperatura utilizada, e atribuíram esse fato ao maior teor de hemiceluloses do bambu em relação às outras biomassas utilizadas. De acordo com Butler et al. (2011), a formação de gases na pirólise de gramíneas e palhas é mais acentuada, uma vez que essas biomassas normalmente possuem altos teores de hemiceluloses.
A casca de arroz, no entanto, apresentou alto teor de lignina, baixo teor de holocelulose e elevado teor de cinzas, levando a uma menor perda de massa das amostras, maior rendimento em subproduto sólido e menor rendimento em condensáveis.

Em relação à composição da fração condensável, em média, $50 \%$ dos gases condensáveis é composta de água (Figura 3). Em um processo industrial, onde haja interesse na valorização química dos condensáveis da torrefação, é desejável que o teor de água na fração condensável seja reduzido, portanto, é necessário estudar os fatores relacionados à formação de água durante o processo. Wannapeera et al. (2011) observaram um 
incremento do teor de água na fração condensável de $6,1 \%$ para $9,5 \%$ quando aumentaram de 0 para 30 minuto o tempo de residência da torrefação da madeira de leucena. Desse modo, utilizar tempos de residência curtos pode ser uma alternativa para reduzir o teor de água na fração condensável do processo de torrefação.

\section{Composição da biomassa e rendimento em condensáveis}

A correlação de Pearson entre as variáveis estudadas é apresentada na Tabela 2. Observou-se a ocorrência de correlações positivas e significativas entre o rendimento em condensáveis e os teores de materiais voláteis e holocelulose e uma correlação negativa e significativa entre o rendimento em condensáveis e o teor de cinzas das biomassas. Não foram observadas correlações significativas entre o rendimento em condensáveis e a composição química elementar das biomassas analisadas.

A correlação positiva entre o teor de materiais voláteis e o rendimento em condensáveis mostrou-se coerente com estudos desenvolvidos na faixa de temperatura da pirólise, como o de Oasmaa et al. (2010), que encontraram correlação positiva entre o rendimento em bioóleo e teor de materiais voláteis das biomassas. Chen et al. (2012) mencionam que a formação de alcatrão durante a pirólise pode estar relacionada à quantidade de materiais voláteis da biomassa.

Nos processos de pirólise rápida, o teor de cinzas e os seus componentes desempenham um papel importante nos rendimentos e na qualidade dos produtos gerados, influenciando negativamente a produção de bioóleo (Oasmaa et al., 2010; Tröger et al., 2012). No presente estudo, durante o processo de torrefação das biomassas, essa influência dos materiais minerais também foi observada. Quanto maior a quantidade de materiais minerais na biomassa, menor o rendimento em condensáveis obtido.

A correlação positiva entre o rendimento em condensáveis e o teor de holocelulose parece refletir o efeito do teor de hemiceluloses das biomassas, uma vez que na faixa de temperatura da torrefação, as hemiceluloses, principal fonte de voláteis, são os polissacarídeos mais degradados (Prins et al., 2006; Arias et al., 2008).

A influência da composição das biomassas analisadas no rendimento em gases condensáveis é mostrada na Figura 4. Observa-se pela figura que a produção de condensáveis na torrefação é favorecida por altos teores de holocelulose e materiais voláteis e baixos teores de cinzas na matéria-prima.

Tabela 2. Correlação de Pearson para rendimento em condensáveis totais (RC), teores de materiais voláteis (MV), cinzas $(\mathrm{CZ})$, carbono fixo (CF), holocelulose (HOL), lignina total (LIG), extrativos solúveis em etanol/tolueno (EXT), carbono (C), hidrogênio $(\mathrm{H})$, nitrogênio $(\mathrm{N})$ e oxigênio $(\mathrm{O})$.

\begin{tabular}{lccccccccccc}
\hline & $\mathbf{R C}$ & $\mathbf{M V}$ & $\mathbf{C Z}$ & $\mathbf{C F}$ & $\mathbf{H O L}$ & $\mathbf{L I G}$ & $\mathbf{E X T}$ & $\mathbf{C}$ & $\mathbf{H}$ & $\mathbf{N}$ & $\mathbf{O}$ \\
\hline $\mathbf{R C}$ & 1 & $0,92^{*}$ & $-0,94^{*}$ & 0,08 & $0,89^{*}$ & $-0,43$ & 0,61 & 0,52 & $-0,15$ & $-0,48$ & $-0,25$ \\
$\mathbf{M V}$ & & 1 & $-0,96^{*}$ & $-0,11$ & $0,97^{*}$ & $-0,73$ & 0,73 & 0,49 & $-0,18$ & $-0,33$ & $-0,26$ \\
$\mathbf{C Z}$ & & & 1 & $-0,19$ & $-0,98^{*}$ & 0,61 & $-0,75$ & $-0,70$ & $-0,08$ & 0,18 & 0,48 \\
$\mathbf{C F}$ & & & & 1 & 0,07 & 0,39 & 0,10 & 0,73 & 0,87 & 0,51 & $-0,78$ \\
HOL & & & & & 1 & $-0,71$ & 0,83 & 0,67 & 0,05 & $-0,12$ & $-0,47$ \\
LIG & & & & & & 1 & $-0,51$ & $-0,17$ & 0,22 & 0,00 & 0,10 \\
$\mathbf{E X T}$ & & & & & & & 1 & 0,75 & 0,30 & 0,21 & $-0,65$ \\
$\mathbf{C}$ & & & & & & & & 1 & 0,75 & 0,46 & $-0,95^{*}$ \\
$\mathbf{H}$ & & & & & & & & & 1 & 0,85 & $-0,90^{*}$ \\
$\mathbf{N}$ & & & & & & & & & & 1 & $-0,70$ \\
\hline
\end{tabular}




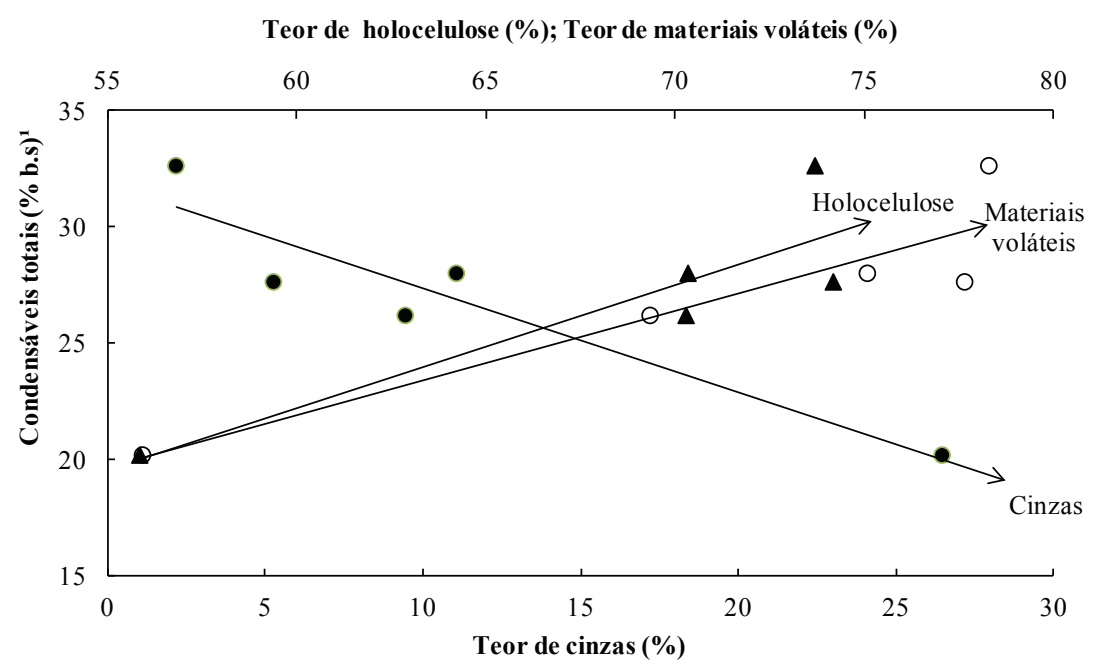

Figura 4. Relações entre o rendimento em condensáveis totais e os teores de holocelulose, materiais voláteis e cinzas das biomassas estudadas. ${ }^{1}$ Base seca.

\section{Conclusões}

A composição da biomassa exerce um importante papel nos rendimentos dos produtos da torrefação. Os teores de holocelulose e materiais voláteis das biomassas analisadas apresentaram correlação positiva e significativa, com o rendimento em gases condensáveis formados no processo de torrefação. Os teores de cinzas das biomassas testadas impactaram negativamente o rendimento em condensáveis da torrefação, apresentando correlação negativa e significativa com este subproduto. As espécies de maior rendimento em condensáveis foram, em ordem decrescente, bambu, bagaço de cana-de-açúcar, capim elefante, casca de pinhão manso e casca de arroz.

\section{Referências}

ALMEIDA, G.; BRITO; J. O.; PERRÉ, P. Alterations in energy properties of eucalyptus wood and bark subjected to torrefaction: the potential of mass loss as a synthetic indicator. Bioresource Technology, Essex, v. 101, p. 9778-9784, 2010.

ARIAS, B.; PEVIDA, C.; FERMOSO, J.; PLAZA, M. G.; RUBIERA, F.; PIS, J. J. Influence of torrefaction on the grindability and reactivity of woody biomass. Fuel Processing Technology, Amsterdam, v. 89, p. 169-175, 2008.

ASSOCIAÇÃO BRASILEIRA DE NORMAS TÉCNICAS. NBR 8112/83: carvão vegetal: análise imediata. Rio de Janeiro, 1986. 6 p.

BUTLER, E.; DEVLIN, G.; MEIER, D.; MCDONNELL, K. A review of recent laboratory research and commercial developments in fast pyrolysis and upgrading. Renewable and Sustainable Energy Reviews, v. 15, p. 4171-4186, 2011.
CHEN, H.; WANG,Y.; XU, G.; YOSHIKAWA, K. Fuel-N evolution during the pyrolysis of industrial biomass wastes with high nitrogen content. Energies, Paris, v. 5, p. 5418-5438, 2012.

CHEN, W.-H.; CHENG, W.-Y.; LU, K.-M.; HUANG, Y.-P. An evaluation on improvement of pulverized biomass property for solid fuel through torrefaction. Applied Energy, London, v. 88, p. 3636-3644, 2011.

DI BLASI, C. Modeling and simulation of combustion processes of charring and non-charring solid fuels. Progress in Energy and Combustion Science, Oxford, v. 19, p. 71 104, 1993.

EHRMAN, T. Determination of acid-soluble lignin in biomass. Kansas: Laboratory Analytical Procedure 004, Midwest Research Institute, $1996.7 \mathrm{p}$.

EUROPEAN COMMITTEE FOR STANDARDIZATION. XP CEN/ TS 15104: 2011: Solid biofuels: determination of total content of carbon, hydrogen and nitrogen: instrumental methods, 2011. 12 p.

FELFLI, F. E. F.; LUENGO, C. A.; SOLER, P. B. Torrefação de biomassa: características, aplicações e perspectivas. In: ENCONTRO DE ENERGIA NO MEIO RURAL, 3., 2000, Campinas. [Anais...] Campinas: AGRENER, 2000. 5 p.

KIM, Y.-H.; LEE; S.-M.; LEE, H.-W.; LEE, J.-W., Physical and chemical characteristics of products from the torrefaction of yellow poplar (Liriodendron tulipifera). Bioresource Technology, Essex, v. 116, p.120-125, 2012.

LEE, J.-W.; KIM, Y.-H.; LEE, S.-M.; LEE, H.-W. Optimizing the torrefaction of mixed softwood by response surface methodology for biomass upgrading to high energy density. Bioresource Technology, Essex, v. 116, p. 471-476, 2012.

MEDIC, D.; DARR, M.; POTTER, B.; SHAH, A. Effect of torrefaction process parameters on biomass feedstock upgrading. In: ANNUAL INTERNATIONAL MEETING BY ASABE, 2010. Pittsburgh. Papers... Pittsburgh: ASABE, 2010. 17 p. Paper n. 1009316. 
OASMAA, A.; SOLANTAUSTA, Y.; ARPIAINEN, V.; KUOPPALA, E.; SIPILÄ, K. Fast pyrolysis bio-oils from wood and agricultural residues. Energy \& Fuels, Washington, US, v. 24, p. 1380-1388, 2010.

OHLIGER, A.; FÃRSTER, M.; KNEER, R. Torrefaction of beechwood: a parametric study including heat of reaction and grindability. Fuel, London, v. 104, p. 607-613, 2013.

OLIVEIRA, J. B.; VIVACQUA FILHO, A.; MENDES, M. G.; GOMES, P. A. Produção de carvão vegetal: aspectos técnicos. In: FUNDAÇÃO CENTRO TECNOLÓGICO DE MINAS GERAIS/ CETEC. Produção e utilização de carvão vegetal. Belo Horizonte, 1982. 1 v. p. 60-89. (Série de Publicações Técnicas, 8).

PÉREZ, J. M. M.; CORTEZ, L. A. B.; GOMEZ, E. O. Pirólise lenta unidimensional de uma grande partícula de capim elefante e bagaço de cana-de-açúcar em um reator de leito fixo. In: ENCONTRO DE ENERGIA NO MEIO RURAL, 4., 2002, Campinas. Proceedings... Disponível em: $<\mathrm{http} / / / \mathrm{www}$.proceedings. scielo.br/scielo.php?script $=$ sci_arttext\&pid=MSC00000000220020 00200038\&lng=en\&nrm=abn>. Acesso em: 15 jul. 2012.

PRINS, M. J.; PTASINSKI, K. J.; JANSSEN, F. J. J. G. Torrefaction of wood: part 2. analysis of products. Journal of Analytical and Applied Pyrolysis, Amsterdam, v. 77, p. 35-40, 2006.

RODRIGUES, T. O.; ROUSSET, P. Effects of torrefaction on energy properties of Eucalyptus grandis wood. Cerne, Lavras, v. 15, p. 446-452, 2009.

ROUSSET, P.; MACEDO, L.; COMMANDRÉ, J. M.; MOREIRA, A. Biomass torrefaction under different oxygen concentrations and its effect on the composition of the solid by-product. Journal of Analytical and Applied Pyrolysis, Amsterdam, v. 96, p. 86-91, 2012.

SEYE, O.; CORTEZ, L. A. B.; GÓMEZ, E. O. Estudo cinético da biomassa a partir de resultados termogravimétricos. In: ENCONTRO DE ENERGIA NO MEIO RURAL, 3., 2000, Campinas. Proceedings... Disponível em: $<$ http://www.proceedings. scielo.br/scielo.php?script $=$ sci_arttext\&pid=MSC00000000220000 00200022\&lng=en\&nrm=abn>. Acesso em: 15 jul. 2012.

TECHNICAL ASSOCIATION OF THE PULP AND PAPER INDUSTRY. T-204 om-88: solvent extractives of wood and pulp: TAPPI Test Methods. Atlanta, 1996a.
TECHNICAL ASSOCIATION OF THE PULP AND PAPER INDUSTRY. T-211 om-93: ash in wood, pulp, paper and paperboard: combustion at $525^{\circ} \mathrm{C}$ :. TAPPI Test Methods. Atlanta, $1996 \mathrm{~b}$.

TECHNICAL ASSOCIATION OF THE PULP AND PAPER INDUSTRY. T-264 om-88: preparation of wood for chemical analysis: TAPPI Test Methods. Atlanta, 1996c.

TEMPLETON, D.; EHRMAN, T. Determination of acid-insoluble lignin in biomass: chemical analysis and testing task. Kansas: Laboratory Analytical Procedure 003, Midwest Research Institute, 1995. $13 \mathrm{p}$.

TRÖGER, N.; RICHTER, D.; STAHL, R. Effect of feedstock composition on product yields and energy recovery rates of fast pyrolysis products from different straw types. Journal of Analytical and Applied Pyrolysis, Amsterdam, v. 100, p. 158-165, 2013.

TUMULURU, J. S.; SOKHANSANJ, S.; HESS, R. J.; WRIGHT, C. T.; BOARDMAN, R. D. A review on biomass torrefaction process and product properties for energy applications. Industrial Biotechnology, Swansea, v. 7, p. 384-401, 2011.

VAMVUKA, D. S.; SFAKIOTAKIS, V. T. Evaluation of production yield and thermal processing of switchgrass as a bio-energy crop for the Mediterranean region. Fuel Processing Technology, Amsterdam, v. 91, p. 988-996, 2010.

WA N N A P E ER A, J .; F U N G TAMM A S A N, B.; WORASUWANNARAK, N. Effects of temperature and holding time during torrefaction on the pyrolysis behaviors of woody biomass. Journal of Analytical and Applied Pyrolysis, Amsterdam, v. 92, p. 99-105, 2011.

WANNAPEERA, J.; WORASUWANNARAK, N. Upgrading of woody biomass by torrefaction under pressure. Journal of Analytical and Applied Pyrolysis, Amsterdam, v. 96, p. 173-180, 2012.

WILD, P. J.; DEN UIL, H.; REITH, J. H.; KIEL, J. H. A.; HEERES, H. J. Biomass valorisation by staged degasification: a new pyrolysisbased thermochemical conversion. Journal of Analytical and Applied Pyrolysis, Amsterdam, v. 85, p. 124-133, 2009.

YAMAN, S. Pyrolysis of biomass to produce fuels and chemical feedstocks. Energy Conversion and Management, Oxford, v. 45, p. 651-671, 2004. 